\title{
The gender-specific expression of neuropeptide $Y$ and neuropeptide $Y$ receptors in human atrial tissue during cardiopulmonary bypass surgery
}

\author{
Fei Meng, Jie Han, Jiangang Wang, Haibo Zhang, Chunlei Xu, Xu Meng \\ Cardiac Valve Center, Department of Cardiac Surgery, Beijing Anzhen Hospital, Capital Medical University, Beijing Institute of Heart Lung and \\ Blood Vessel Diseases, Beijing 100029, China \\ Contributions: (I) Conception and design: F Meng, X Meng; (II) Administrative support: X Meng; (III) Provision of study materials or patients: J Han, \\ X Meng; (IV) Collection and assembly of data: F Meng, J Wang, H Zhang, C Xu; (V) Data analysis and interpretation: F Meng; (VI) Manuscript \\ writing: All authors; (VII) Final approval of manuscript: All authors. \\ Correspondence to: Xu Meng. Cardiac Valve Center, Department of Cardiac Surgery, Beijing Anzhen Hospital, Capital Medical University, No. 2 \\ Anzhen Road, Beijing 100029, China. Email: mxu@263.net.
}

\begin{abstract}
Background: Cardiac sympathetic nervous system is usually activated in cardiopulmonary bypass (CPB) surgery, accompanied by excessive release of norepinephrine (NE). Neuropeptide Y (NPY) has been shown to regulate NE release in the terminal of sympathetic fiber, which is a target for regulating heart function. The expression of NPY and NPY receptor (NPYR) genes in the human atrial tissues during CPB in cardiac surgery was investigated in the present study.

Methods: A few discarded atrial tissues before and after CPB were collected in 22 patients with rheumatic cardiac valve diseases. The transcriptional levels of NPY and NPYRs were monitored by real-time quantitative polymerase chain reaction (RT-qPCR) method. Moreover, the correlation between the mRNA levels of NPY/NPYRs and the clinical data were investigated in detail.

Results: The mRNA levels of NPY Y1 and NPY Y5 genes were statistically attenuated in male patients after CPB. Conversely, the expression of NPY, NPY Y1 and NPY Y5 genes were enhanced in female patients. Correlation analysis suggested that there was a significant negative correlation between cardiac ejection fraction (EF) after CPB with the atrial transcriptional level of NPY in male patients.

Conclusions: These results suggested that the expression of NPY/NPYRs in human atrial tissue during CPB was gender specific and activated NPY signaling was only identified in female patients. The elevated expression level of NPY in male patients was correlated with lower cardiac EF after CPB.
\end{abstract}

Keywords: Neuropeptide Y (NPY); NPY receptor (NPYR); cardiopulmonary bypass (CPB) surgery

Submitted May 03, 2018. Accepted for publication Nov 08, 2018.

doi: $10.21037 /$ jtd.2018.11.126

View this article at: http://dx.doi.org/10.21037/jtd.2018.11.126

\section{Introduction}

Myocardial ischemia-reperfusion (I/R) injury is a wellrecognized pathophysiological process, which occurs in several clinical conditions such as infarction, shock, organ transplantation and cardiopulmonary bypass (CPB) surgery (1). Several pathogenetic mechanisms involved have been explored. Among them, the imbalance between sympathetic and parasympathetic system inputs to the heart, is one of the major reasons which induce myocardial ischemia, arrhythmia and death (2).

Neuropeptide Y (NPY) is a 36-residue peptide amide, costored and co-released with noradrenaline in sympathetic nerve fibers of postganglionic neurons innervating the cardiovascular system. NPY is associated with hypertension (3), myocardial infarction (4), cardiomyopathy (5), suggesting that NPY functions in cardiovascular homeostasis. To 
Table 1 Clinical characteristics of patients

\begin{tabular}{lccc}
\hline Characteristic & All & Male & Female \\
\hline Number & 22 & 9 & 13 \\
Age & $51.77 \pm 2.597$ & $51.78 \pm 4.245$ & $51.77 \pm 3.414$ \\
EF before CPB & $63.14 \pm 1.282$ & $62.00 \pm 1.708$ & $64.00 \pm 1.867$ \\
LVIDd before CPB & $49.71 \pm 1.890$ & $51.22 \pm 3.483$ & $48.58 \pm 2.116$ \\
LVIDs before CPB & $33.00 \pm 1.336$ & $34.11 \pm 2.406$ & $32.17 \pm 1.542$ \\
Total CPB time (m) & $89.45 \pm 8.522$ & $92.33 \pm 12.501$ & $87.46 \pm 11.922$ \\
Ischemic time (m) & $62.27 \pm 5.868$ & $66.56 \pm 11.572$ & $59.31 \pm 6.194$ \\
Reperfusion time $(\mathrm{m})$ & $27.18 \pm 5.175$ & $25.78 \pm 2.778$ & $28.15 \pm 8.693$ \\
EF after CPB & $59.36 \pm 1.754$ & $56.00 \pm 2.205$ & 0.504 \\
LVIDd after CPB & $45.41 \pm 1.372$ & $48.78 \pm 1.722$ & 0.786 \\
LVIDs after CPB & $31.00 \pm 1.208$ & $33.78 \pm 1.470$ & 0.557 \\
\hline
\end{tabular}

Data are presented as $n$ or mean \pm SEM. EF, ejection fraction; CPB, cardiopulmonary bypass; LVIDd, left ventricular internal diastolic diameter; LVIDs, left ventricular internal systolic diameter, SEM, standard error of the mean.

date, six NPY receptors (NPYRs), namely NPYRs Y1-Y6 have been reported $(6,7)$. NPYRs regulate the biological processes through diverse ways. NPY Y1 promoted cellular shortening and calcium transient in rat ventricular myocytes (8). Antagonists of NPY Y2 could prevent the inhibition of vagal bradycardia after sympathetic stimulation (9). The influence of vagal neurotransmission and ventricular myocyte excitability was accomplished even in the presence of $\beta$-receptor blockade, which indicated that pharmacologically targeting NPYRs might be a useful therapeutic strategy synergistically with $\beta$-receptor blockers (10). Moreover, the expression of NPY was increased in human plasma after CPB (11), implying NPY signaling controlled the pathological progression of CPB. However, the expression changes of NPY and NPYRs in human heart itself during CPB surgery remain elusive. Whether the expression of NPY and NPYRs have any correlations with clinical data needs to be investigated.

\section{Methods}

\section{Collection of specimens}

Patients were hospitalized for cardiac surgery in Anzhen Hospital, Beijing. According to preoperative Doppler echocardiography, rheumatic mitral stenosis or insufficiency were found in these patients, partly with tricuspid insufficiency. Patients with cerebrovascular accidents, diabetes mellitus, myocardial infarction and other diseases were excluded. All researches involving human participants were approved by Medical Ethics Committee. Written informed consent was obtained from the patients and data were analyzed anonymously. Patient characteristics were summarized in Table 1. All patients underwent superior vena cava catheterization in the right atrial appendage. A few specimens were taken from the right atrium during the intubation of the superior vena cava (before $\mathrm{CPB}$ ) and the extubation of the superior vena cava (after CPB), which were routinely excised and discarded tissue. The specimens were stored in RNA stabilization reagent (Qiagen) for later analysis.

\section{Total RNA extraction of patients' atrium}

Trizol was used to extract the total RNAs from patients' atrium. Trizol (sigma) was added in patients' atrial tissue for lysis, then chloroform and isopropanol were used for purification, finally the supernatant of centrifugation was dissolved in water with RNase free, and stored at $-80^{\circ} \mathrm{C}$.

\section{Reverse transcription of $m R N A$}

The total mRNA from patients' atrium was reversely transcribed according to SuperScript ${ }^{\mathrm{TM}}$ III RT kit (Invitrogen). Appropriate amount of freshly extracted RNAs with Primer, dNTP mix, SuperScript III RT were used. 
Table 2 qPCR primers

\begin{tabular}{|c|c|c|c|c|}
\hline \multicolumn{2}{|c|}{ Primer name } & \multicolumn{3}{|c|}{ Primer sequence } \\
\hline \multirow{2}{*}{\multicolumn{2}{|c|}{ Human NPY }} & \multicolumn{3}{|c|}{ Forward: AAAACGATCCAGCCCAGAG } \\
\hline & & \multicolumn{3}{|c|}{ Reverse: GCTGAAAATAGGAAAAGGCCAG } \\
\hline \multirow{2}{*}{\multicolumn{2}{|c|}{ Human NPY Y1 }} & \multicolumn{3}{|c|}{ Forward: CAAGCCCAGTCGCATITAAAA } \\
\hline & & \multicolumn{3}{|c|}{ Reverse: CAGGTAATCAAAGTATGTTGCAGG } \\
\hline \multirow{2}{*}{\multicolumn{2}{|c|}{ Human NPY Y2 }} & \multicolumn{3}{|c|}{ Forward: ACATCTTGTTTCCGCGTCTC } \\
\hline & & \multicolumn{3}{|c|}{ Reverse: CCCATTITCAGTACAGGTCCAC } \\
\hline \multirow{2}{*}{\multicolumn{2}{|c|}{ Human NPY Y5 }} & \multicolumn{3}{|c|}{ Forward: CCGAGGTCTGCTCATTGTG } \\
\hline & & \multicolumn{3}{|c|}{ Reverse: TCTAACTITGTGGCAGGTCAG } \\
\hline \multirow{2}{*}{\multicolumn{2}{|c|}{ Human GAPDH }} & \multicolumn{3}{|c|}{ Forward: TGGGTGTGAACCATGAGAAG } \\
\hline & & \multicolumn{3}{|c|}{ Reverse: GAGTCCTTCCACGATACCAAAG } \\
\hline \multicolumn{5}{|c|}{$\begin{array}{l}\text { qPCR, quantitative polymerase chain reaction; NPY, } \\
\text { neuropeptide } Y \text {. }\end{array}$} \\
\hline \multicolumn{5}{|c|}{$\begin{array}{l}\text { Table } 3 \text { The relative mRNA levels of NPY, NPY Y1 and NPY Y5 } \\
\text { genes in patients }\end{array}$} \\
\hline Gene & & All & Male & Female \\
\hline NPY & 1.09 & 50 (4.99) & $0.5500(1.07)$ & $2.1400(10.11)$ \\
\hline$N P Y Y 1$ & 1.03 & $00(3.19)$ & $0.3700(0.56)$ & $2.5100(6.17)$ \\
\hline NPY Y5 & 1.22 & $50(9.70)$ & $0.4300(0.32)$ & $6.6800(20.68)$ \\
\hline
\end{tabular}

NPY, neuropeptide $Y$.

The resulting cDNAs were preserved at $-20^{\circ} \mathrm{C}$.

\section{Real-time quantitative polymerase chain reaction (RT-qPCR)}

RT-qPCR was carried out with SYBR Green (ABI). The total reaction system was $20 \mu \mathrm{L}, 50^{\circ} \mathrm{C} 2 \mathrm{~min}, 95^{\circ} \mathrm{C} 10 \mathrm{~min}$, 40 cycles for $95^{\circ} \mathrm{C} 15 \mathrm{sec}, 60^{\circ} \mathrm{C} 1 \mathrm{~min}$. GAPDH was set as the internal parameter and the relative mRNA levels were calculated with the $2^{-\Delta \Delta \mathrm{Ct}}$ method. The primers were listed in Table 2.

\section{Statistical analysis}

Results were presented as means \pm standard error of the mean (SEM) or median (interquartile range). Data were evaluated by one-way ANOVA or nonparametric tests. A value of $\mathrm{P}<0.05$ was considered statistically significant.

\section{Results}

\section{General information}

Twenty-two patients hospitalized for cardiac surgery due to rheumatic heart disease were randomly enrolled. These patients received cardiac valve replacement surgery by $\mathrm{CPB}$. The mean age of all patients was $51.77 \pm 2.597$, total CBP time was $89.45 \pm 8.522 \mathrm{~min}$, ischemic time was $62.27 \pm 5.868 \mathrm{~min}$, reperfusion time was $27.18 \pm 5.175 \mathrm{~min}$. There was no statistically difference between male and female patients in such general information. The cardiac functions such as ejection fraction (EF), left ventricular internal diastolic diameter (LVIDd), left ventricular internal systolic diameter (LVIDs) were detected by echocardiography before and after CPB. After surgery, the patients' cardiac functions were partly recovered, such as LVIDd returned to normal range (Table 1).

\section{The expression of NPY and NPYRs in buman atrium before and after $C P B$ surgery}

Using RT-qPCR, the transcriptional levels of NPY and NPY receptor genes were analyzed. We found NPY and NPY Y1, Y2, Y5 genes could express in human atrium, of them, the transcriptional level of NPY Y2 was the least. The mRNA levels of NPY, NPY Y1 and NPY Y5 were compared before and after CPB in each patient (Table 3). For all 22 patients, the relative mRNA levels of NPY, NPY $\mathrm{Y} 1, \mathrm{NPY}$ Y5 were 1.0950 (4.99) $(\mathrm{P}=0.236), 1.0300$ (3.19) $(\mathrm{P}=0.069)$ and $1.2250(9.70)(\mathrm{P}=0.149)$ respectively. There was no statistically difference before and after CPB in all patients. But the relative mRNA levels of NPY Y1 [0.3700 (0.56), $\mathrm{P}=0.021]$ and NPY Y5 [0.4300 (0.32), $\mathrm{P}=0.011]$ were statistically down-regulated in male patients after $\mathrm{CPB}$ (Figure 1). On the contrary, the relative transcriptional levels of NPY [2.1400 (10.11), P=0.039], NPY Y1 [2.5100 (6.17), $\mathrm{P}=0.019$ ] and NPY Y5 [6.6800 (20.68), $\mathrm{P}=0.004$ ] were statistically up-regulated in female patients after $\mathrm{CPB}$ (Figure 2). These results implied that NPY and NPYRs might play a role in CPB surgery, although the mechanism could be different between male and female patients.

\section{Correlation analysis}

The correlation between patients' clinical cardiac functions (LVIDd, LVIDs, EF before and after CPB), CPB 


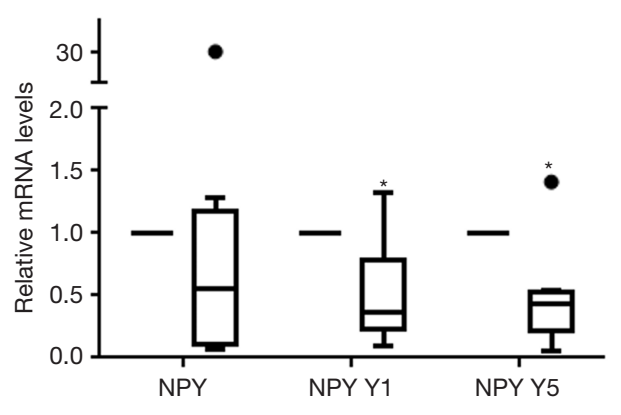

Figure 1 The transcriptional levels of NPY, NPY Y1 and NPY Y5 genes after $\mathrm{CPB}$ were compared with before $\mathrm{CPB}$ in male patients. Using qPCR, the relative mRNA levels of NPY Y1 and NPY Y5 were statistically down-regulated after $\mathrm{CPB}$. *, $\mathrm{P}<0.05$. NPY, neuropeptide Y; CPB, cardiopulmonary bypass; qPCR, quantitative polymerase chain reaction.

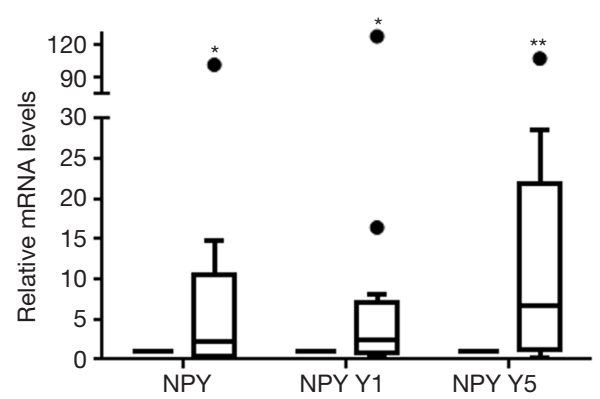

Figure 2 The transcriptional levels of NPY, NPY Y1 and NPY $\mathrm{Y} 5$ genes after $\mathrm{CPB}$ were compared with before $\mathrm{CPB}$ in female patients. Using qPCR, the relative mRNA levels of NPY, NPY Y1 and NPY Y5 were statistically up-regulated after $\mathrm{CPB}$. * $\mathrm{P}<0.05$, **, $\mathrm{P}<0.01$. NPY, neuropeptide $\mathrm{Y}$; CPB, cardiopulmonary bypass; qPCR, quantitative polymerase chain reaction.

surgery time (ischemia time, reperfusion time), and the transcriptional levels of NPY, NPY Y1, NPY Y5 were all analyzed. There was only a significant negative correlation between cardiac EF after $\mathrm{CPB}$ and the relative mRNA levels of NPY (Spearman's correlation coefficient $=-0.706$, $\mathrm{P}=0.034)$ in male patients. There was no correlation in other factors.

\section{Discussion}

CPB needs to block the cardiac blood flow first, myocardial oxygenation is restored after myocardial ischemia for a short period, and ischemia reperfusion injury happens simultaneously (1). In this process, the cardiac function is regulated by the autonomic nervous system. It is generally accepted that the activated sympathetic system and the suppressed parasympathetic system contribute to the increased blood pressure, myocardial oxygen consumption and cerebrovascular accidents (2). The sympathetic system mainly releases norepinephrine (NE) and activated $\beta$ adrenergic receptors to increase heart rate and myocardial contraction. Antagonistically, the parasympathetic system release acetylcholine $(\mathrm{ACh})$ and activated muscarinic ACh receptors on the opposite way.

It has been proved that NPY regulates NE release in the terminal of sympathetic fibers. The NPY signaling plays comprehensive roles in all typed cardiac cells. In human right ventricular endocardial endothelial cells, NPY Y1 modulates the cytosolic and nuclear calcium homeostasis, and their secretion of NPY (12). In cardiomyocytes, NPY had been shown to stimulate hypertrophy of rat and chick cardiomyocytes through activation of NPY Y1 receptor $(13,14)$. Pellieux et al. found that NPY via NPY Y5 receptor participated in the development of mouse cardiac hypertrophy through MAPK cascade (15). These data suggest NPY Y1 and Y5 have positive chronotropic effects on ventricular cardiomyocytes. With respect to NPY Y2 receptor, it reduces ACh release and vagal bradycardia after sympathetic stimulation (16). These data mentioned above suggest that pharmacologically targeting NPYRs might conduce to cardiovascular homeostasis.

To our knowledge, this is the first reports that the transcriptional levels of NPY and NPYRs genes were investigated in atrial samples from patients with rheumatic diseases before and after CPB surgery. Using qPCR, we found that NPY, NPY Y1, NPY Y2 and NPY Y5 were all expressed in human right atrium, which was consistent with Ejaz's study (17). But the NPY Y2 transcriptional level was so hard to detect in our patients. Since NPY Y2 receptor was thought to be a presynaptic receptor (18), its transcriptional level was little.

In all 22 patients, there was no statistically difference between the transcriptional levels of NPY, NPY Y1 and NPY Y5 before and after CPB. In spite of the sex factor, our data revealed that NPY mRNA level was up-regulated after CPB in female patients. It had been reported that patient plasma NPY concentration was elevated after CPB since cardiac sympathetic nerve activity was increased after surgery (19). In this study the up-regulation of NPY mRNA in female patients in atrium might be attributed to the elevated NPY levels in plasma. In addition, we found that, only four people had atrial fibrillation after $\mathrm{CPB}$ in 
our 22 patients, and interestingly they were all females. Guler group demonstrated that NPY played a role in the occurrence of atrial fibrillation in the patients after coronary bypass surgery (11). Hence, the atrial fibrillation in female patients might be associated with up-regulation of NPY expression. Since the number of atrial fibrillation patients was small, this manifestation needed to be investigated further. Though there was no statistically difference between the transcriptional levels of NPY in male patients before and after $\mathrm{CPB}$, we found that the relative mRNA level of NPY was negative correlated with cardiac EF after $\mathrm{CPB}$ in these patients. Luo et al. reported that there was a dose-dependent stimulation of NPY with decreased ATP content and activity in the rat cardiomyocytes (20). Thus, our findings reinforced that the decreased level of NPY mRNA in male atriums might help the patients to recover cardiac functions after surgery.

In this study, the transcriptional levels of NPY Y1 and NPY Y5 were both up-regulated in female patients, but down-regulated in male patients after $\mathrm{CPB}$, which suggested that NPY signaling was activated in female patients. Jackson et al. found that NPY Y1 expression level was higher in the male rat skeletal muscle compared with that in females (21). In our atrium muscle tissue, the expression levels of NPY Y1 and NPY Y5 were also stronger in male patients than that of female patients. Musso et al. found that estradiol could stimulate NPY Y1 transcription through estrogen receptor alpha (ER- $\alpha$ ) (22). However, we could not find significant difference in the mRNA levels of ER- $\alpha$ in female patients before and after CPB (data not shown). Since ER- $\alpha$ moved from cytoplasm to nucleus to regulate target genes (23), the up-regulation of NPY Y1 expression in female patients might be due to the cytoplasm-nucleus translocation. NPY and its receptors had been found gender-specificity in other disease, such as type 2 diabetes (24) and severe periodontitis (25). Since targeting NPYRs pharmacologically may be a useful therapeutic strategy synergistically with $\beta$-blockers for cardiovascular diseases, the gender-specificity should be taken into account.

\section{Conclusions}

Our results suggested that there was gender-specify in the expression of NPY and NPY receptor genes during CPB surgery and NPY signaling was activated in female patients. Gender differences should be considered when using NPYRs as pharmacologically targets for the treatment of cardiovascular diseases.

\section{Acknowledgements}

We thank Professor Zhiqing Xu, Professor Liang Li from the Capital Medical University; Professor Xuejun Jiang from Chinese Academy of Sciences for their supports.

\section{Footnote}

Conflicts of Interest: The authors have no conflicts of interest to declare.

Ethical Statement: The study was approved by the ethics review board of the Capital Medical University affiliated Beijing Anzhen Hospital (No. 2009001X) and written informed consent was obtained from all patients.

\section{References}

1. Niemann B, Schwarzer M, Rohrbach S. Heart and Mitochondria: Pathophysiology and Implications for Cardiac Surgeons. Thorac Cardiovasc Surg 2018;66:11-9.

2. Finlay M, Harmer SC, Tinker A. The control of cardiac ventricular excitability by autonomic pathways. Pharmacol Ther 2017;174:97-111.

3. Baltazi M, Katsiki N, Savopoulos C, et al. Plasma neuropeptide Y (NPY) and alpha-melanocyte stimulating hormone (a-MSH) levels in patients with or without hypertension and/or obesity: a pilot study. Am J Cardiovasc Dis 2011;1:48-59.

4. Cuculi F, Herring N, De Caterina AR, et al. Relationship of plasma neuropeptide $\mathrm{Y}$ with angiographic, electrocardiographic and coronary physiology indices of reperfusion during ST elevation myocardial infarction. Heart 2013;99:1198-203.

5. Dvorakova MC, Kruzliak P, Rabkin SW. Role of neuropeptides in cardiomyopathies. Peptides 2014;61:1-6.

6. Wahlestedt C, Grundemar L, Hakanson R, et al. Neuropeptide Y receptor subtypes, Y1 and Y2. Ann N Y Acad Sci 1990;611:7-26.

7. Lecklin A, Lundell I, Paananen L, et al. Receptor subtypes Y1 and Y5 mediate neuropeptide Y induced feeding in the guinea-pig. Br J Pharmacol 2002;135:2029-37.

8. Heredia MP, Delgado C, Pereira L, et al. Neuropeptide $\mathrm{Y}$ rapidly enhances $[\mathrm{Ca} 2+] \mathrm{i}$ transients and $\mathrm{Ca} 2+$ sparks in adult rat ventricular myocytes through $\mathrm{Y} 1$ receptor and PLC activation. J Mol Cell Cardiol 2005;38:205-12.

9. Smith-White MA, Hardy TA, Brock JA, et al. Effects of a selective neuropeptide Y Y2 receptor antagonist, 
BIIE0246, on Y2 receptors at peripheral neuroeffector junctions. Br J Pharmacol 2001;132:861-8.

10. Herring N. Autonomic control of the heart: going beyond the classical neurotransmitters. Exp Physiol 2015;100:354-8.

11. Guler N, Ozkara C, Dulger H, et al. Do cardiac neuropeptides play a role in the occurrence of atrial fibrillation after coronary bypass surgery? Ann Thorac Surg 2007;83:532-7.

12. Jacques D, D'Orléans-Juste P, Magder S, et al. Neuropeptide $\mathrm{Y}$ and its receptors in ventricular endocardial endothelial cells. Can J Physiol Pharmacol 2017;95:1224-9.

13. Millar BC, Schluter KD, Zhou XJ, et al. Neuropeptide $\mathrm{Y}$ stimulates hypertrophy of adult ventricular cardiomyocytes. Am J Physiol 1994;266:C1271-7.

14. Jacques D, Abdel-Samad D. Neuropeptide Y (NPY) and NPY receptors in the cardiovascular system: implication in the regulation of intracellular calcium. Can J Physiol Pharmacol 2007;85:43-53.

15. Pellieux C, Sauthier T, Domenighetti A, et al. Neuropeptide Y (NPY) potentiates phenylephrine-induced mitogen-activated protein kinase activation in primary cardiomyocytes via NPY Y 5 receptors. Proc Natl Acad Sci U S A 2000;97:1595-600.

16. Herring N, Lokale MN, Danson EJ, et al. Neuropeptide $\mathrm{Y}$ reduces acetylcholine release and vagal bradycardia via a Y2 receptor-mediated, protein kinase C-dependent pathway. J Mol Cell Cardiol 2008;44:477-85.

17. Ejaz A, LoGerfo FW, Khabbaz K, et al. Expression of Neuropeptide Y, Substance P, and their receptors in the right atrium of diabetic patients. Clin Transl Sci

Cite this article as: Meng F, Han J, Wang J, Zhang H, Xu $\mathrm{C}$, Meng X. The gender-specific expression of neuropeptide $\mathrm{Y}$ and neuropeptide $\mathrm{Y}$ receptors in human atrial tissue during cardiopulmonary bypass surgery. J Thorac Dis 2018;10(12):6563-6568. doi: 10.21037/jtd.2018.11.126
2011;4:346-50.

18. Smith-White MA, Herzog H, Potter EK. Role of neuropeptide Y Y(2) receptors in modulation of cardiac parasympathetic neurotransmission. Regul Pept 2002;103:105-11.

19. Cooper TJ, Clutton-Brock TH, Jones SN, et al. Factors relating to the development of hypertension after cardiopulmonary bypass. Br Heart J 1985;54:91-5.

20. Luo G, Xu X, Guo W, et al. Neuropeptide Y damages the integrity of mitochondrial structure and disrupts energy metabolism in cultured neonatal rat cardiomyocytes. Peptides 2015;71:162-9.

21. Jackson DN, Milne KJ, Noble EG, et al. Gendermodulated endogenous baseline neuropeptide Y Y1receptor activation in the hindlimb of Sprague-Dawley rats. J Physiol 2005;562:285-94.

22. Musso R, Maggi A, Eva C. 17beta-estradiol stimulates mouse neuropeptide $\mathrm{Y}-\mathrm{Y}(1)$ receptor gene transcription by binding to estrogen receptor alpha in neuroblastoma cells. Neuroendocrinology 2000;72:360-7.

23. Sebastian T, Sreeja S, Thampan RV. Import and export of nuclear proteins: focus on the nucleocytoplasmic movements of two different species of mammalian estrogen receptor. Mol Cell Biochem 2004;260:91-102.

24. Campbell CD, Lyon HN, Nemesh J, et al. Association studies of BMI and type 2 diabetes in the neuropeptide $\mathrm{Y}$ pathway: a possible role for NPY2R as a candidate gene for type 2 diabetes in men. Diabetes 2007;56:1460-7.

25. Freitag-Wolf S, Dommisch H, Graetz C, et al. Genomewide exploration identifies sex-specific genetic effects of alleles upstream NPY to increase the risk of severe periodontitis in men. J Clin Periodontol 2014;41:1115-21. 